\title{
A Foilized Polyethylene Pouch for the Prevention of Transmission of HIV from Mother to Child
}

\author{
Robert Malkin* and Caroline Howard
}

Department of Biomedical Engineering, Hudson 136, \#90281, Duke University, Durham, NC 27708, USA

\begin{abstract}
Many children become HIV+ due to mother-to-child transmission, a risk that can be largely eliminated if infants ingest antiretroviral (ARV) medications immediately after birth. As most mothers in Africa deliver at home, the ARV must be provided at their last antenatal visit, sometimes months before birth. No current drug delivery system allows the mother to store the medication at home long enough to be effective. We propose a preserving, foilized, polyethylene pouch to be pre-dosed and sealed by a pharmacist for later delivery to the newborn.

Pouches were filled with $0.6 \mathrm{ml}$ of Nevirapine (NVP). Thirty-three pouches were immediately studied to measure the impact of medication handling (oxygen, light, filling and sealing the pouches). The remaining samples were stored for up to one year at three storage conditions $\left(25^{\circ} \mathrm{C} / 60 \% \mathrm{RH}, 30^{\circ} \mathrm{C} / 65 \% \mathrm{RH}\right.$, and $\left.40^{\circ} \mathrm{C} / 75 \% \mathrm{RH}\right)$. Every two months, moisture loss, preservative concentration, impurity concentrations and NVP concentration were measured. Flora and fauna challenges were conducted.

The pouch nearly eliminated moisture loss: pouches lost less than $0.7 \%$ of their weight over twelve months. As expected, exposing the medication to light, oxygen, and handling significantly affected the sacrificial preservative concentrations (Propyl paraben dropped 38\%, Methyl paraben dropped 12\% at time point zero). However, after the initial time point, preservative levels were stable in the package over twelve months under all storage conditions $(4.1 \%$ average concentration drop), leaving sufficient preservatives to protect the medication. The concentration of NVP changed an average of only $1.3 \%$ over all storage conditions and times points (maximum $1.4 \%$ ).

We conclude that the foilized polyethylene pouch can preserve NVP, and perhaps other ARV's, for up to one year.
\end{abstract}

Keywords: HIV, PMTCT, anti-retroviral preservation, home birth.

\section{INTRODUCTION}

The vast majority of the 390,000 new HIV cases in children in 2010 were attributed to mother-to-child transmission [1]. Transmission can happen in the womb, during birth or as a result of breast feeding. Transmission during birth and breastfeeding can be prevented by giving the child antiretroviral therapy immediately after birth and during the breastfeeding period.

But children are not getting the treatment they need. Children living in resource poor settings are a particularly difficult population to reach. Forty-five percent of HIV+ mothers receive ARV (antiretroviral) treatment, while less than $33 \%$ of children born to HIV+ mothers receive prophylactic antiretroviral drugs [2]. Much of the difficulties that are unique to children are related to the technical challenges of the preferred treatment regimes.

Some public health organizations recommend that infants receive a small dose of a liquid antiretroviral such as Nevirapine (NVP) oral suspension immediately after birth

\footnotetext{
*Address correspondence to this author at the Department of Biomedical Engineering, Hudson 136, \#90281, Duke University, Durham, NC 27708, USA; Tel: +1919-660-8266; Fax: 919-684-4488;
}

E-mail: robert.malkin@duke.edu and daily for either six weeks or until one week after the breastfeeding period ends [3]. The infants should receive their first dose within 72 hours of birth to be effective, and preferably within 24 hours. In Sub-Saharan Africa, many clinics possess these medications but cannot easily distribute them to the large percentage of mothers who give birth at home. For example, according to the United Nations Population Fund, 57\% of all Tanzanian women delivered at home in 2005 [4].

While the mother could travel to a clinic immediately after birth to obtain the ARV, an HIV+ mother will often be reluctant to travel the day or two after delivering her baby. She may not have funds or means for transport to a hospital, feel too weak to travel to a clinic miles away, or fear stigmatization after explaining why she must go to a clinic after delivering an apparently healthy baby.

If the mother might deliver at home and might not return to the clinic in time, it is logical to provide the infant's ARVs in advance. Unfortunately, it is not possible to provide infant antiretrovirals, like NVP, many months before delivery for several reasons. Infants cannot swallow pills or tablets. Pharmacists in resource-poor settings have been packaging single doses in oral syringes, cups, and other ad hoc solutions [5] but these have very limited shelf lives, and they are prone to contamination. An improved solution 


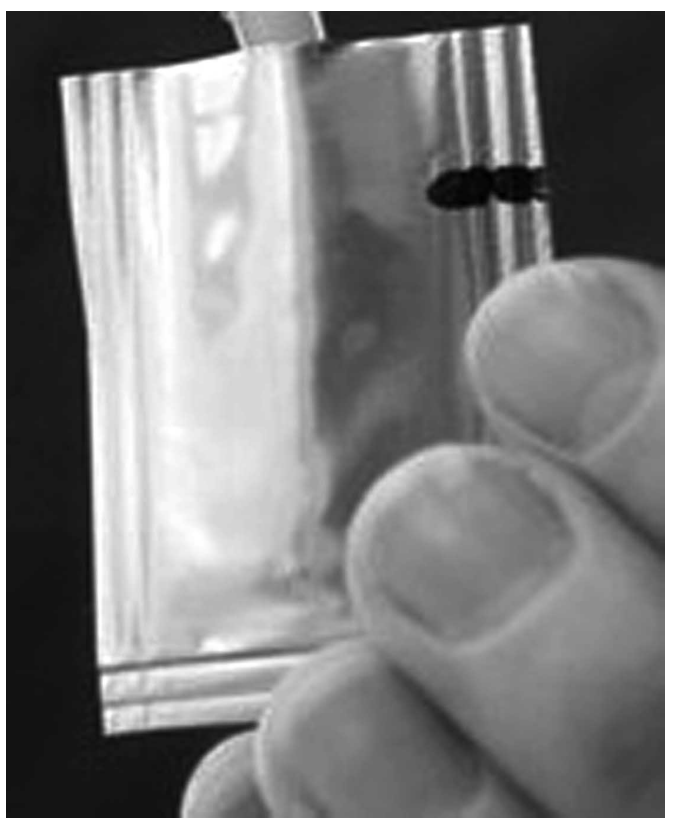

Fig. (1). This small and inexpensive pouch can be easily torn open by mothers. The pouch can store liquid antiretrovirals for twelve months, allowing mothers delivering at home to prevent HIV transmission to their infants.

places the syringe in a protective pouch such as the ExactaMed pre-dosed oral syringe adhesive sealed foil bag developed by PATH [Seattle, WA, USA]. However, the oral syringes may leak and the removable tip may be a choking hazard. Even with the protective pouch, the drug is only predicted to last two months, perhaps due to moisture and preservative loss. Two months is far less than the maximum of many months that can elapse between maternal diagnosis and delivery. And, in resource-poor settings, a mother's first antenatal visit may be her last before delivery. Manufactures have been unwilling to release a single dose package because of the cost of development and regulatory approval and the limited market outside the developing world.

Thus, at this moment, there is an unmet need for a drug delivery system to deliver ARVs to HIV+ pregnant mothers months before they deliver for administration to their children in the event that they deliver at home. This paper describes a new solution to the problem based on a foilized, polyethylene single-dose pouch (Fig. 1); a solution that has been widely used in the food industry for many years [7].

\section{METHODS}

Large foilized polyethylene material (PAKVF4, outside polyester/ polyethylene with a 0.00035 inches thick Al foil layer and an inside linear low density polyethylene layer, 4.3 mil total thickness, Sorbent Systems, Los Angeles, CA) were cut into rectangles and sealed to yield pouches with final inner dimensions of 1.0 by 2.5 inches. An electric heat sealer (Model IPKHS-606T, Impak Corporation, Los Angeles, CA) was used to seal three sides of the pouches with a seal width of approximately 0.5 inches. After being allowed to heat up for ten minutes, the sealer was applied for ten seconds for each seal. Pouches were visually inspected to ensure adequate seals on each of the three sides. Pouches were serialized and weighed before further processing. Pouches were not sterilized or cleaned.
Pouches were then filled by hand with $0.6 \mathrm{~mL}$ NVP oral suspension $(10 \mathrm{mg} / \mathrm{mL}$, Boehringer Ingelheim) using a 3.0 $\mathrm{mL}$ blunted needle syringe. Pouches were closed with the sealing method described above immediately after filling and then weighed again. Two bottles of NVP were used to fill all of the pouches. The bottle used and each bottle's manufacturer lot number and expiry date were noted for each pouch.

\section{Stability Testing}

Two-hundred and ninety-nine NVP-filled pouches were submitted to the Southern Testing and Research Division of Microbac Laboratories, Inc. (Wilson, NC) for twelve months of controlled storage and testing. Samples were stored in walk in stability chambers at $25^{\circ} \mathrm{C} / 60 \%$ relative humidity $(\mathrm{RH})$ and $40^{\circ} \mathrm{C} / 75 \% \mathrm{RH}$ and in a reach in chamber at $30^{\circ} \mathrm{C} / 65 \%$ RH. The selected temperature and humidity ranges are expected to represent extremes of African home storage and match the US Food and Drug Administration (FDA) recommended testing conditions [6, 8]. Storage conditions did not fall outside of $+/-2^{\circ} \mathrm{C}$ and $+/-5 \% \mathrm{RH}$ of marked values. Each pouch was stored at $5^{\circ} \mathrm{C}$ after being pulled until it was tested.

Microbiological, weight change, and chemical testing was performed on the pouches. Microbiological testing was performed at time zero and after 12 months. Flora and fauna challenges (total microbial aerobic, yeast and mold) were attempted on twenty pouches at each storage condition at the start of the experiment and on another twenty pouches after twelve months of storage following US Pharmacopeia part 61 (USP61) using USP33-NF28 methodology. (The United States Pharmacopeia (USP) and The National Formulary (NF) is a book of public pharmacopeia standards for the testing of chemical and biological drug substances.) Ten pouches at each storage condition were tested for the presence of E. Coli (USP62) at the start of the experiment and retested on another ten pouches after twelve months of storage also following USP33-NF28 methodology.

Samples were weighed prior to stability testing and again when pulled at months $2,4,6,8,10$, and 12, and weight loss or gain was used to estimate moisture loss during the stability storage.

Lastly, chemical testing was performed at months 2, 4, 6, $8, \quad 10$, and 12 following a modified USP32-NF-27 methodology. The modifications to the standard procedures were 1) use of the bulk NVP pulled from the original bottles (stored at $5^{\circ} \mathrm{C}$ ) for the preparation of method standards at time points 2, 4, 6, 8 and 10 (time points zero and twelve months were completed with USP NVP reference standard as per USP32-NF27 without modification), and 2) a reduced sample volume. Specifically, the three pouches were combined, and approximately 0.4 g-aliquots of test samples were volumetrically diluted to $10.0 \mathrm{~mL}$ (refer to monograph procedure) to yield working solutions at a NVP concentration of about $400 \mu \mathrm{g} / \mathrm{mL}$ (nominal). For each instrumental set-up, the system suitability was established prior to samples analysis, and continued system suitability was monitored following the USP method. All samples were analyzed by high performance liquid chromatography (HPLC) with duplicate injections for NVP assay, related compounds and impurities determination. Duplicates were combined to form single data points according to USP32. 
Table 1. Summary of 2009 Field Acceptability Study Responses

\begin{tabular}{|c|l|}
\hline \multicolumn{2}{|l|}{ Nurse Sampling } \\
\hline \hline 6 & Total hospitals represented \\
\hline 10 & Total number providers interviewed \\
\hline Nurse Interview Results \\
\hline $90 \%$ & $\begin{array}{l}\text { Thought mothers could effectively tear open pouch and drop } \\
\text { medicine into child's mouth without training }\end{array}$ \\
\hline $100 \%$ & $\begin{array}{l}\text { Thought most mothers could effectively administer drug to } \\
\text { their infant if trained how to use the pouch by provider }\end{array}$ \\
\hline $50 \%$ & $\begin{array}{l}\text { Had concerns about using the device to distribute NVP (e.g. } \\
\text { stigma, lack of training/education leading to noncompliance } \\
\text { such as the use of intermediary apparatus like a spoon or cup) }\end{array}$ \\
\hline
\end{tabular}

In addition to determination of the NVP assay, levels of Methyl paraben and Propyl paraben (preservatives) in the samples were estimated using independently prepared standards. This was performed at all measured time points. The standards were prepared at $50 \mu \mathrm{g} / \mathrm{mL}$ for Methyl paraben and at $10 \mu \mathrm{g} / \mathrm{mL}$ for Propyl paraben.

The chromatographs were analyzed to determine if impurities were induced in the medication or extracted from the plastic $[9,10]$. To determine the origin of the impurities, two additional analyses were made: preservatives by themselves and NVP by itself. Methyl and Propyl paraben standard solutions were prepared in water/methanol mixedsolvent and were stored in clear glass containers at ambient temperature for four months. USP NVP reference standard was prepared according the USP $32-\mathrm{NF} 27$ methodology and analyzed.

Three pouches were combined for each measurement of NVP assay. Twenty pouches were used for each USP61 measurement and ten for each USP62 measurement. Because the assay testing was completed at each of seven time points and USP61 and USP62 were each completed twice, the total number of pouches required for testing was 243 . The remaining 56 were prepared but never used.

For the NVP assay and related compound data, each reported data point represents the analysis of three sample pouches and multiple data points combined, as required by USP testing. Since, only one data point results, no statistical analysis can be performed on any one individual time point and test condition.

As a comparison, thirty syringes were filled with water then placed in pouches of identical material to that described above. After sealing with double-sided adhesive tape, the pouches were weighed and moved to a storage compartment at room temperature. Pouches were removed and weighed approximately every other day for twenty days.

\section{User Acceptability Testing}

To determine the acceptability of the pouch among healthcare workers and end users in Sub-Saharan Africa, ten nurses in six hospitals in northern Tanzania were interviewed. All participating nurses interviewed worked directly with HIV+ mothers in their hospital's maternity ward and were familiar with NVP and prevention of motherto-child transmission (PMTCT) national protocols. Three questions were asked, as shown in Table $\mathbf{1 .}$

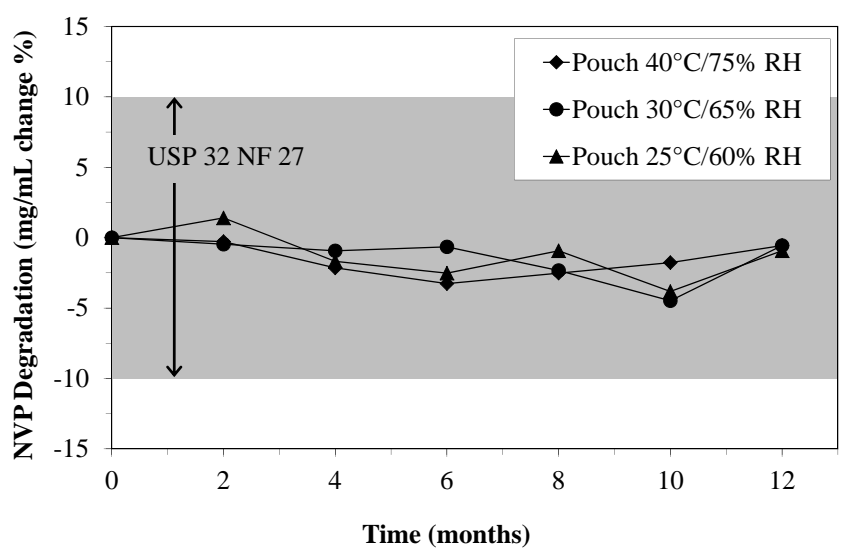

Fig. (2). The percent degradation of NVP oral suspension fell well within the USP 32 NF 27 standard even after twelve months in the pouch.

As a more objective measure of the volunteers' ability to open the pouches, tear time was measured by asking ten volunteers in the United States to open the pouches. The tear time was measured as the time from when the pouch was handed to the volunteer until the volunteer felt they had opened the pouch. Participants were provided with two pouches filled with placebo doses of the same viscosity as NVP and with picture instructions with minimal text. No verbal instructions were provided. Participants were asked to empty the pouches into a small cup (to simulate giving the infant liquid medication). If the participant indicated that the picture instructions were unclear, the participant was provided with the same picture instructions with the addition of one to three word instructional captions in English (for example, "tear").

\section{RESULTS}

\section{Stability Testing}

The most critical function of the pouch is to preserve the active ingredient for later delivery to the infant. Fig. (2) shows that the NVP concentrations remained nearly unchanged for all time points at all storage conditions. The average change of the NVP was observed to be a $1.36 \%$ drop (maximum 1.40\%) in the concentration of NVP when compared to the bottle stored at $5^{\circ} \mathrm{C}$ during the same time period. This is well within the 10\% USP acceptability standard. In other words, the pouch preserves the medication adequately for up to twelve months.

Because we used the USP reference standard at time points zero and twelve, we can also determine how well the manufacturer's bottle can store NVP. The original material was $106.8 \%$ of the specified $10 \mathrm{mg} / \mathrm{mL}$ marked on the bottle. After twelve months of storage, the bottle dropped to $102.9 \%$ of the specified $10 \mathrm{mg} / \mathrm{mL}$. Again, these values are well within the USP standard $(+/-10 \%)$.

At time zero and twelve months later, the pouches passed all microbial challenges. E. coli was absent. The aerobic and yeast counts were both below $10 \mathrm{cfu} / \mathrm{mL}$, which is well below the acceptance criteria for the tests. In summary, the testing demonstrated a complete absence of any sort of biological or microbial contamination despite having sealed the pouches in conditions typical of a pharmacist, not a sterile medical processing facility. 


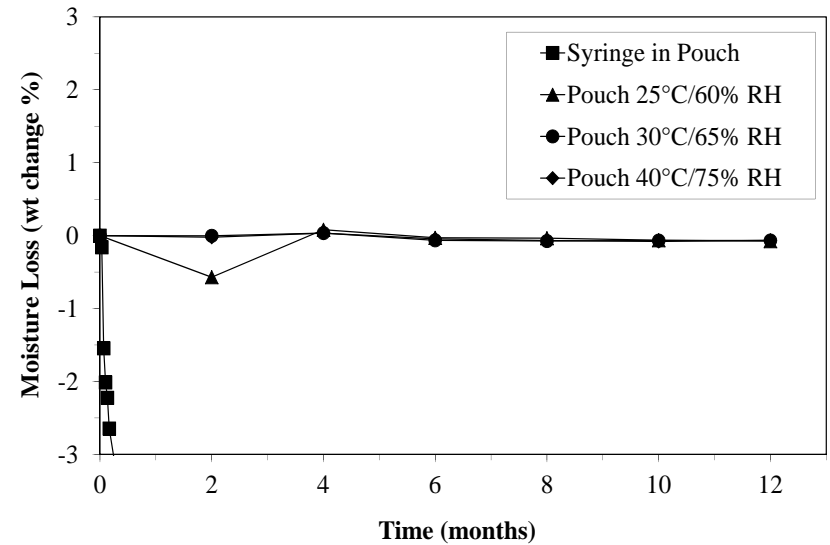

Fig. (3). Moisture loss may have limited the shelf life of NVP oral suspension stored in a syringe for two months, but when stored in the pouch, the medication looses almost no moisture even after twelve months.
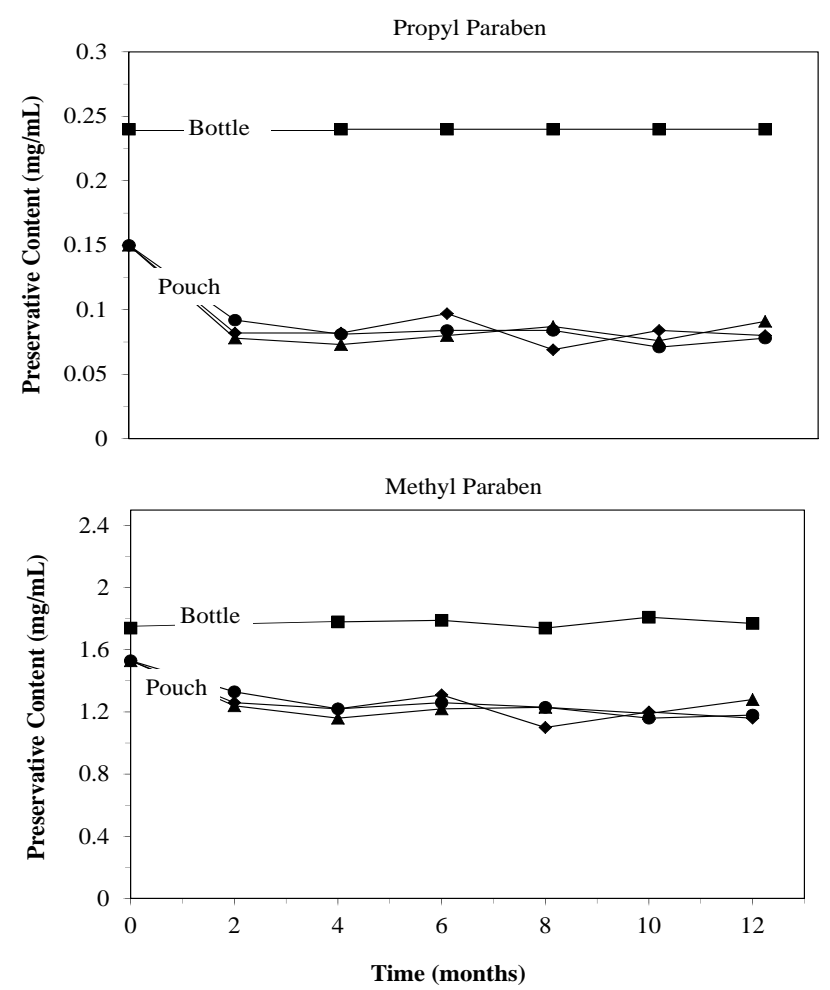

Fig. (4). Parabens act as sacrificial oxidants. Their loss may limit shelf life. Despite initial losses attributed to filling and sealing, the sacrificial oxidants remained in adequate quantities to preserve the medication $\left(\boldsymbol{\Delta} 25^{\circ} \mathrm{F} / 60 \% \mathrm{RH}: \bullet 30^{\circ} \mathrm{F} / 65 \% \mathrm{RH}:-40^{\circ} \mathrm{F} / 75 \% \mathrm{RH}\right)$.

Infant NVP is held in an aqueous suspension by emulsification agents. As mentioned earlier, we suspected that, when stored in a syringe or a syringe inside a foil pouch, moisture loss quickly concentrated the NVP beyond usability. As shown in Fig. (3), when NVP is stored in the pouch, it loses less than $0.7 \%$ of its weight over twelve months. In the syringe, moisture is lost well beyond tolerable limits in less than two months (Fig. 3). The pouch nearly eradicated moisture loss.

Handling the medication, including exposure to light, oxygen and mechanical treatment can damage the medication. As already presented above, we did not see any evidence of this in the concentrations of NVP. However, there are other ways to determine if material handling affected the medication, specifically preservatives and impurities. Fig. (4) does show that some preservatives were lost during the filling and sealing of the pouches (time point zero). Propyl paraben dropped $38 \%$, and Methyl paraben dropped $12 \%$ initially. However, after the initial time point, preservative levels were stable in the package over twelve months under all storage conditions $(4.1 \%$ average concentration drop after initial drop). The initial drop could be due to the medicines exposure to heat, oxygen or light when it is drawn into the syringe but probably occurred within minutes or hours of repackaging. The bag itself can not be the cause, as Fig. (4) shows no loss of preservatives after the initial stabilization period. The lack of significant change in NVP levels and lack of microbial growth suggest that the preservatives did act as sacrificial oxidants, leaving sufficient preservatives to protect the medication for twelve months.

There were five impurities noted in the samples from the bottle upon opening (time point zero), the stored bulk samples from the bottle $\left(5^{\circ} \mathrm{C}\right.$, time point twelve months) and the stored samples in the pouch (all storage conditions and time points). The same five impurities were found in all three: the freshly opened bottle, the stored bottle and the stored pouches. All impurities were present at concentrations below $1 \%$ in all samples stored under all conditions. Storage in the pouch did not introduce any impurities that were not already present in the original material analyzed when the bottles were first opened. This strongly suggests that the pouch does not induce impurities in the medication and the medication does not extract impurities from the pouch (or at least none that are not also extracted from the manufacturer's bottle).

For this discussion purposes we arbitrarily number the impurities from the highest to the lowest relative retention times. Of the five impurities, USP reference standard NVP shows impurity four. Therefore, we conclude that this is a breakdown product of NVP. Aged samples of the preservatives show impurities two and five. Therefore, we conclude that these are breakdown products of the preservatives. The breakdown products of these chemicals were studied and their safety documented in the original manufacturers submission to the FDA. This leaves impurities one and three.

Impurities one and three were present in very small quantities $(<0.3 \%)$ in all samples including recently opened bottles, stored bottles and samples stored in pouches. Therefore, we conclude that storage in the pouch poses no additional risk over storage in the bottle. However, we can gain some additional insight from our data. Impurities one and three seem to be most dramatically affected by handling, as they increased from $<0.02 \%$ to 0.04 and $0.06 \%$ at time point zero. However, storage and storage conditions did affect their concentrations $(0.05$ and $0.08 \%$ at four months for $25 \mathrm{C} / 60 \%$ and 0.11 and $0.18 \%$ at four months for $40 \mathrm{C} / 75 \%$ ). Nevertheless, storage for twelve months at extremes of temperature and humidity created only as much of impurities one and three as would handling the medication approximately four times $(0.19 \%$ and $0.29 \%$ at twelve months $40 \mathrm{C} / 75 \%$ ). The bag itself is probably not involved in the creation of impurities one and three as storage for twelve months at $25 \mathrm{C} / 60 \%$ created just barely measurable 
additional concentrations of the impurities over those present at time point zero $(0.06 \%$ and $0.12 \%$ at 12 months)

\section{User Acceptability Testing}

Nearly all (90\%) of the ten healthcare workers (nurses) thought mothers could empty the pouch contents into their infants mouths with pictures alone and no training (Table 1). All $(100 \%)$ nurses felt that with proper training mothers could empty the pouch properly at home. About half of the nurses expressed concerns about using the device, mostly relating to the HIV stigma (the risk of revealing one's status by using the pouch) and to a sound understanding of pouch use. For example, one nurse was worried that despite an instructional consultation, a mother may squeeze the drug into a contaminated cup or spoon before giving to the infant, which may cause diarrhea.

The engineering study in the US indicated that end users can easily tear open the pouch without a cutting device. No failures to open the pouch were observed during the tear time study. The overall average time to tear the pouch open was $1.98 \mathrm{~s}$ with a standard deviation of $1.28 \mathrm{~s}$.

\section{DISCUSSION}

The immediate target population for the ARV pouch includes mothers who are already receiving ARVs but whose infants might not have access to ARVs for post exposure prophylaxis immediately after birth. The World Health Organization (WHO) notes that in 2008, 45\% of mothers living with HIV received ARVs for PMTCT compared to only $32 \%$ of infants, leaving a "critical" $13 \%$ gap in ARV coverage [2]. The report also notes that in the same year, $77 \%$ of mothers came into a hospital or clinic for one or more antenatal care visits [2]. The data presented here suggests that the pouch can fill this gap. Mothers can be given a pouch containing ARVs for their infant at initial diagnosis, then wait until delivery, perhaps many months later, to provide the ARV to their infant. The active ingredient will still be available in the pouch in its original concentration without contamination, and mothers will likely be able to deliver it to their infants. Thus, our data suggests that the pouch will have a significant impact on HIV transmission to children.

The pouch was first intended to package infant Nevirapine. However, national PMTCT protocols continue to change. One advantage of the pouch is that it is made of the same materials used to bottle nearly all ARVs. Therefore, we suspect that the pouch will perform equally well for nearly all ARVs. We have begun testing Zidovudine (AZT) and Lamivudine (3TC). Unlike other approaches to the provision of ARVs to children, the pouch, as it is sealed at the pharmacy, can be quickly adapted to new ARVs and to new protocols using existing ARVs.

Even for ARV protocols with multiple doses of multiple medications, the small size and weight of the package makes it relatively easy for the mother to maintain privacy concerning her status.

The pouch can be used after birth as well. The pouch, since it is sealed by a pharmacist, can facilitate a regimen tailored to a particular population, a particular experiment or even a particular patient's mass, health or status.
There are continuing efforts to decentralize treatment access points. With the pouch, other healthcare workers such as traditional birthing assistants (TBAs) or health-post workers could be employed to reach rural infants' PMTCT needs (specifically infant NVP and infant AZT/3TC) immediately after birth and until mothers can return to the hospital or clinic for their first post natal care visit. Under this scenario, the pouch would be sealed under the guidance of pharmacists in centralized or near-centralized compounding facilities. Giving TBAs prescription medications in circumstances requiring urgent provision is not a novel concept. Currently in Guatemala, Nepal, and other low-resource settings, ministries of health permit TBAs to administer oxytosin and misoprostol for home birthing mothers, to minimize postpartum hemorrhaging during child birth. TBAs also give antibiotics to prevent blindness to rural populations.

The price and versatility of the pouch make it an attractive solution. The pouch can be manufactured for US $\$ 0.04$ when one million pouches are ordered. It can be obtained for less in larger orders. Pharmacists also require a commercially available electric sealer, estimated at US $\$ 120$, to seal pouches after filling. Due to the long shelf life of the pouch, it is possible that a pharmacist would fill and seal large batches of pouches in a large urban hospital or centralized compounding facility. These pouches could be provided to patients at pharmacies and dispensaries in more rural communities or where demand for the medication is low and shelf life of open bottles a challenge.

The extended shelf life of medications in the pouch would help mitigate frequent stock outs and supply chain variability commonly encountered with essential medicines in disaster response situations and in rural settings where disease prevalence may be low. The pouch may also be a cheap and versatile alternative to pill bottles, tissue paper bags, syringes, and reused vials for packaging pills in low resource settings, both rural and urban.

In the United States, the foilzed, polyethylene pouch is already FDA approved for short-term storage of liquid medications. Essentially, the FDA considers the pouch a pill bottle for liquids. The FDA's industry guidance document for short term liquid medication storage vessels states that "a patient's exposure to substances extracted from plastic packaging ... is expected to be comparable to a patient's exposure to the same substances through the use of the same material when used to package food" [5]. Indeed, juice boxes, wine, ketchup and many other foods are already packaged in foilized polyethylene containers made from the same materials as that proposed here. Therefore, no additional regulatory considerations are required for the suggested application in the US. Many foreign countries recognize FDA approval. Therefore, no additional regulatory considerations are required for many countries in the world.

\section{LIMITATIONS}

We did not have HIV mothers take the medication home and deliver it to their infants. There may be problems that are not simulated with our nursing interviews or US engineering studies. American volunteers tearing packages may not be a representative population of African HIV+ mothers with respect to dexterity, education, and familiarity with opening 
such pouches. We compared the pouch to samples pipetted from a bottle of NVP stored at $5^{\circ} \mathrm{C}$. In other words, we compared the performance of the pouch to near perfect storage and extraction conditions. In actual clinical use, the bottle might have been opened many times before a given patient receives their dose and would have been stored in warmer, uncontrolled conditions. Furthermore, the dose may be held in the syringe for many hours or may be prepared in a spoon for the patient. Had we compared the pouch to more realistic storage and delivery conditions our results would have been even more favorable to storage in the pouch.

\section{CONCLUSION}

In conclusion, the need for a new technology for antiretroviral HIV medication delivery is met by the foilized polyethylene pouch described here. The pouch can safely store NVP for up to twelve months. Unlike any other available solution, the pouch can be immediately and economically deployed to reach infant populations at great risk of contracting HIV.

\section{CONFLICT OF INTEREST}

The author confirms that this article content has no conflicts of interest.

\section{ACKNOWLEDGEMENT}

This work supported by PEPFAR and OAR supplemental funding to NIH grant number 5U01-AI069484-05.

\section{REFERENCES}

[1] UN. UNAIDS Data Tables, USA: United Nations 2010

[2] WHO, UNAIDS, UNICEF. Towards universal access: scaling up priority HIV/AIDS interventions in the health sector. Progress Report 2009. Geneva, Switzerland: WHO 2009; p. 112.

[3] WHO, 2010. Antiretroviral drugs for treating pregnant women and preventing HIV infection in infants: Towards Universal Access. Geneva, Switzerland: WHO Publication 2010.

[4] UNFPA. The Prime Minister of Tanzania Calls on Regional Leaders to Increase Health Spending to 15 Per Cent of National Budgets and to Strive for Achieving Health MDGs. 2007. Available at: http://www.unfpa.org/public/News/pid/308

[5] Berman A, Brooke S. Sourcing guide: the nevirapine infant-dose pouch for use in prevention of mother-to-child transmission of hiv/aids programs. Version 1. Seattle, Washington: PATH 2006; 2. Available at: http://www.path.org/files/TS_NVP_sourcing_guide.pdf

[6] FDA. Guidance for industry: container closure systems for packaging human drugs and biologic. USA: CMC May, 1999 Section III.F.1

[7] Brown W. plastics in food packaging. New York: Dekker 1992

[8] ICH. Guidance for Industry. Q1A(R2) Stability Testing of New Drug Substances and Products. Rev 2. U.S: US. Department of Health and Human Services, Food and Drug Administration, November 2003

[9] Félix JS, Isella F, Bosetti O, Nerín C. Analytical tools for identification of non-intentionally added substances (NIAS) coming from polyurethane adhesives in multilayer packaging materials and their migration into food simulants. Anal Bioanal Chem 2012; 403(10): 2869-82

[10] Peychès-Bach A, Dombre C, Peyron S, Moutounet M, Chalier P. Effect of ethanol on the sorption of four targeted wine volatile compounds in a polyethylene film. J Agric Food Chem 2012. [Epub ahead of print].

Received: March 03, 2012

(C) Malkin and Howard; Licensee Bentham Open.

This is an open access article licensed under the terms of the Creative Commons Attribution Non-Commercial License (http://creativecommons.org/licenses/by-nc/3.0/) which permits unrestricted, non-commercial use, distribution and reproduction in any medium, provided the work is properly cited. 